\title{
Nuclear spin circular dichroism
}

Juha Vaara, Antonio Rizzo, Joanna Kauczor, Patrick Norman and Sonia Coriani

\section{Linköping University Post Print}

\section{Tweet}

N.B.: When citing this work, cite the original article.

Original Publication:

Juha Vaara, Antonio Rizzo, Joanna Kauczor, Patrick Norman and Sonia Coriani, Nuclear spin circular dichroism, 2014, Journal of Chemical Physics, (140), 13, 134103.

http://dx.doi.org/10.1063/1.4869849

Copyright: American Institute of Physics (AIP) http://www.aip.org/

Postprint available at: Linköping University Electronic Press http://urn.kb.se/resolve?urn=urn:nbn:se:liu:diva-106854 


\section{AD| $\begin{aligned} & \text { The Journal of } \\ & \text { Chemical Physics }\end{aligned}$}

\section{Nuclear spin circular dichroism}

Juha Vaara, Antonio Rizzo, Joanna Kauczor, Patrick Norman, and Sonia Coriani

Citation: The Journal of Chemical Physics 140, 134103 (2014); doi: 10.1063/1.4869849

View online: http://dx.doi.org/10.1063/1.4869849

View Table of Contents: http://scitation.aip.org/content/aip/journal/jcp/140/13?ver=pdfcov

Published by the AIP Publishing

\section{Articles you may be interested in}

Magnetic circular dichroism of symmetry and spin forbidden transitions of high-spin metal ions

J. Chem. Phys. 113, 5003 (2000); 10.1063/1.1289531

Magnetic circular dichroism of spinforbidden transitions in the Fe3+ highspin system

J. Chem. Phys. 59, 1732 (1973); 10.1063/1.1680256

Theory of Magnetic Circular Dichroism

J. Chem. Phys. 52, 3489 (1970); 10.1063/1.1673514

Vibronic Theory of Circular Dichroism

J. Chem. Phys. 51, 984 (1969); 10.1063/1.1672167

Vibrational Structuring in Circular Dichroism

J. Chem. Phys. 42, 2244 (1965); 10.1063/1.1696275

\section{AlP Re-register for Table of Content Alerts}




\title{
Nuclear spin circular dichroism
}

Juha Vaara, ${ }^{1, a)}$ Antonio Rizzo, ${ }^{2}$ Joanna Kauczor, ${ }^{3}$ Patrick Norman, ${ }^{3}$ and Sonia Coriani ${ }^{4, b)}$

${ }^{1}$ NMR Research Group, Department of Physics, University of Oulu, P.O. Box 3000, FIN-90014 Oulu, Finland

${ }^{2}$ Istituto per i Processi Chimico-Fisici del Consiglio Nazionale delle Ricerche (IPCF-CNR),

Area della Ricerca, via G. Moruzzi 1, I-56124 Pisa, Italy

${ }^{3}$ Department of Physics, Chemistry and Biology, Linköping University, S-58183 Linköping, Sweden

${ }^{4}$ Dipartimento di Scienze Chimiche e Farmaceutiche, Università degli Studi di Trieste, Via L. Giorgieri 1, I-34127 Trieste, Italy

(Received 5 February 2014; accepted 18 March 2014; published online 3 April 2014)

\begin{abstract}
Recent years have witnessed a growing interest in magneto-optic spectroscopy techniques that use nuclear magnetization as the source of the magnetic field. Here we present a formulation of magnetic circular dichroism (CD) due to magnetically polarized nuclei, nuclear spin-induced CD (NSCD), in molecules. The NSCD ellipticity and nuclear spin-induced optical rotation (NSOR) angle correspond to the real and imaginary parts, respectively, of (complex) quadratic response functions involving the dynamic second-order interaction of the electron system with the linearly polarized light beam, as well as the static magnetic hyperfine interaction. Using the complex polarization propagator framework, NSCD and NSOR signals are obtained at frequencies in the vicinity of optical excitations. Hartree-Fock and density-functional theory calculations on relatively small model systems, ethene, benzene, and 1,4-benzoquinone, demonstrate the feasibility of the method for obtaining relatively strong nuclear spin-induced ellipticity and optical rotation signals. Comparison of the proton and carbon-13 signals of ethanol reveals that these resonant phenomena facilitate chemical resolution between non-equivalent nuclei in magneto-optic spectra. () 2014 AIP Publishing LLC. [http://dx.doi.org/10.1063/1.4869849]
\end{abstract}

\section{INTRODUCTION}

Natural optical activity arises in chiral media due to their asymmetric interaction with the magnetic component of electromagnetic radiation. ${ }^{1}$ By applying an external magnetic field $\boldsymbol{B}_{0}$, optical activity can be observed in materials regardless of their chirality. ${ }^{2}$ Classical magneto-optic phenomena such as the Faraday rotation of linearly polarized light propagating along $\boldsymbol{B}_{0},{ }^{3}$ the ellipticity induced at spectral regions close to optical excitations in magnetic circular dichroism (MCD), ${ }^{4}$ or (in the Voigt setup with $\boldsymbol{B}_{0} \perp \hat{\boldsymbol{k}}$, the direction of propagation of the light beam) the induced linear birefringence of the Cotton-Mouton effect, ${ }^{5}$ have been known for a long time. Theoretical analysis of these effects involves nonlinear interactions between the electromagnetic beam, $\boldsymbol{B}_{0}$, and the electronic wave function of the system in question. ${ }^{1,6-8}$

As shown in 2006 by Savukov, Lee, and Romalis, ${ }^{9}$ magneto-optic effects can also be caused by the field due to the collective magnetization of nuclei, such as typically used in nuclear magnetic resonance experiments. In Ref. 9, nuclear spin-induced optical rotation (NSOR) was observed in the Faraday set-up for liquid water and xenon. Later work has demonstrated, using a multi-pass cavity apparatus, the existence of an optical chemical shift, the differing NSOR angles caused by nuclear spin polarization in different molecular liquids. ${ }^{10}$ This phenomenon was predicted using first-

\footnotetext{
a)juha.vaara@iki.fi

b) coriani@units.it
}

principles calculations according to the response theory formulation of the underlying antisymmetric polarizability. ${ }^{6}$ Further experimental ${ }^{11-13}$ and theoretical ${ }^{14-16}$ work has been reported for NSOR. Nuclear spin- and electric quadrupole moment-induced Cotton-Mouton effects have been theoretically investigated in Refs. 17-21.

The predicted $^{6}$ resolution of the chemically nonequivalent sites of identical nuclei in a molecule, akin to the high-resolution conventional nuclear magnetic resonance spectrum of ethanol of $1951,{ }^{22}$ has not yet been experimentally observed in nuclear magneto-optic spectroscopy (NMOS). The antisymmetric polarizability underlying NSOR increases rapidly as the incident light frequency approaches optical excitations, ${ }^{23-25}$ which has been proposed to facilitate a means of gaining chemical resolution of the nuclear magneto-optic rotation signals arising from the different chromophores of the molecule. ${ }^{6}$ The first-principles NSOR calculations carried out so far ${ }^{6,10,15,16}$ have all been performed with conventional quadratic response theory, ${ }^{26}$ disregarding the fact that the perturbational approach is not valid in the vicinity of the excitation energies. Hence, it remains a question whether the conclusions drawn in the earlier work remain valid in a more realistic treatment of the near-resonant spectral regions.

Formally the nuclear magneto-optic observables may be obtained by replacing the interaction of the electron cloud with the external magnetic field occurring in the classical magneto-optic phenomena by the corresponding hyperfine interactions with the magnetic moments of the nuclei. ${ }^{6,9,17-21}$ By analogy, also the conventional MCD effect may be 
generalized to nuclear spin-induced circular dichroism (NSCD), where the nuclear magnetization causes a differential absorption of left- and right-circularly polarized light, expressible via induced elliptic polarization onto the incident linearly polarized light. ${ }^{1}$ Whereas the MCD ellipticity provides information on the excitations occurring in the entire molecule, and may be used for analytic purposes such as, e.g., to distinguish different fullerenes as shown in recent work, ${ }^{27}$ NSCD furnishes a nuclear site-specific observable that may, at least in principle, be used to obtain a high-resolution spectrum. The ubiquitous Faraday $\mathcal{B}$ term contribution to MCD, arising from magnetic mixing of the excited states, ${ }^{2}$ has in the past been obtained in electronic structure calculations by the analytical residues of appropriate quadratic response functions $^{28}$ (QRFs) or magnetic-field derivatives of transition strengths, ${ }^{29,30}$ sometimes combined with empirical lineshape functions to simulate the spectral profiles. In addition, both finite-magnetic-field ${ }^{31}$ and sum-over-states perturbation theory (Ref. 32 and references therein) have been employed. The Faraday $\mathcal{A}$-term, also contributing in highsymmetry closed-shell systems, involves orbital degeneracy of the excited states ${ }^{2}$ and requires using complex molecular orbitals. $^{33,34}$

Norman and co-workers ${ }^{35,36}$ have proposed a complex polarization propagator (CPP) approach where a wellbehaving and straightforward response theory treatment of near-resonant phenomena is facilitated by introducing (in the QRFs) a single empirical linewidth parameter $\gamma$ to account for the finite lifetime of the excited states. In this method, the optical rotation (dispersion) angle arising in the normal Faraday rotation or NSOR experiment is obtained as the imaginary part of the relevant QRF, whereas the MCD ellipticity can be directly calculated from the corresponding real part. ${ }^{37}$ Thus, the CPP approach eliminates the need to evaluate the separate $\mathcal{A}$ and $\mathcal{B}$ terms in MCD calculations of closed-shell systems. ${ }^{2,38}$ By the analogy between conventional and nucleus-induced magneto-optic observables mentioned above, the CPP method should be applicable for rigorous investigations of NSOR at light frequencies in the neighborhood of optical excitations as well as NSCD, albeit involving the $\gamma$ parameter. In the past, the CPP method has been used to investigate a variety of molecular properties such as natural optical activity ${ }^{39} \mathrm{x}$-ray absorption, ${ }^{40}$ dispersion forces, ${ }^{41}$ two-photon absorption, ${ }^{42}$ and MCD. ${ }^{27,37,38}$ MCD calculations using related damped response theory approaches have also been performed by Krykunov et al. ${ }^{43}$ and by Kjærgaard et al. ${ }^{34}$ Ref. 44 reported recently a real-time density-functional theory (DFT) method for calculating both the $\mathcal{A}$ and $\mathcal{B}$ terms.

In this paper, we predict the existence of NSCD and formulate expressions of its observable ellipticity, using the analogy between magneto-optic effects caused by an external magnetic field and the field from an ensemble of spinpolarized nuclei. We employ the CPP approach to calculate using Hartree-Fock (HF) and DFT methods the NSCD ellipticity for the nuclei of ethene $\left(\mathrm{C}_{2} \mathrm{H}_{4}\right)$, benzene $\left(\mathrm{C}_{6} \mathrm{H}_{6}\right)$, and para-benzoquinone ( $p \mathrm{BQ}, \mathrm{C}_{6} \mathrm{H}_{4} \mathrm{O}_{2}$ ). We illustrate the similarities and differences in the information that can be extracted from the nucleus-specific, local NSCD spectroscopy (for nuclei of different kind and in different molecules) as opposed to the global MCD method. We demonstrate using calculations of ethanol $\left(\mathrm{CH}_{3} \mathrm{CH}_{2} \mathrm{OH}\right)$ that similar nuclei at chemically non-equivalent sites in the same molecule produce distinct features in the NSCD spectrum, which facilitates high-resolution spectroscopy. In addition, we investigate the behavior of the NSOR angle in the optical absorption region using the non-divergent CPP methodology, to verify the earlier suggestion ${ }^{6}$ of enhanced NSOR signal in such conditions.

\section{THEORY}

The antisymmetric dynamic dipolar polarizability of a molecule $\left(\alpha_{\epsilon \tau}^{\prime}=-\alpha_{\tau \epsilon}^{\prime}\right.$, dependence on the circular frequency $\omega$ implied) may be expanded as a Taylor series in terms of the small magnetic interactions arising from the external magnetic field $\boldsymbol{B}_{0}$ and nuclear magnetic moments $\boldsymbol{m}_{K}=\gamma_{K} \hbar \boldsymbol{I}_{K}$, where $\gamma_{K}$ and $\boldsymbol{I}_{K}$ are the gyromagnetic ratio and spin vector of nucleus $K$, as ${ }^{6,17,23}$

$$
\alpha_{\epsilon \tau}^{\prime}=\sum_{\nu} \alpha_{\epsilon \tau, \nu}^{\prime(B)} B_{v}+\sum_{\nu} \alpha_{\epsilon \tau, v}^{\prime\left(I_{K}\right)} I_{K, \nu}+\mathcal{O}\left(B_{0}^{3}, I_{K}^{3}\right),
$$

$\epsilon \tau \nu$ being the Cartesian coordinates in the molecule-fixed frame. $\boldsymbol{\alpha}^{\prime}$ vanishes in the absence of magnetic fields.

Consider an experiment in which linearly polarized light beam of angular frequency $\omega$ travels along the laboratory $Z$ axis $(\boldsymbol{k} \| \hat{\boldsymbol{Z}})$ through a path of length $l$ in a medium consisting of isotropically tumbling molecules, the number density of which is $\mathcal{N}$. In these conditions, the beam acquires an elliptical polarization with the ellipticity parameter $\eta$, as well as undergoes an optical rotation through angle $\phi$, obtained as ${ }^{1}$

$$
\begin{aligned}
& \eta=\frac{1}{2} \omega \mu_{0} c_{0} l \mathcal{N} \Re\left\langle\alpha_{X Y}^{\prime}\right\rangle, \\
& \phi=\frac{1}{2} \omega \mu_{0} c_{0} l \mathcal{N} \Im\left\langle\alpha_{X Y}^{\prime}\right\rangle,
\end{aligned}
$$

where $c_{0}$ is the speed of light in vacuo, $\mu_{0}$ is the vacuum permeability, and the angular brackets \langle\rangle denote isotropic rotational averaging.

\section{A. Magnetic circular dichroism}

In a MCD measurement, an external magnetic field $\boldsymbol{B}_{0}=B_{0} \hat{\boldsymbol{Z}}$ is oriented along the direction of light propagation, rendering the isotropic average of the antisymmetric polarizability $^{1}$

$$
\left\langle\alpha_{X Y}^{\prime}\right\rangle=\frac{1}{6} B_{0} \sum_{\epsilon \tau \nu} \varepsilon_{\epsilon \tau \nu} \alpha_{\epsilon \tau, \nu}^{\prime\left(B_{0}\right)},
$$

where the Greek subscripts now denote coordinates in the molecule-fixed frame and $\varepsilon_{\epsilon \tau \nu}$ is the Levi-Civita tensor. Hence, the observable MCD ellipticity and OR angle per unit path length and magnitude of the external magnetic field 
strength become ${ }^{37}$

$$
\begin{aligned}
& \frac{\eta^{\left(B_{0}\right)}}{l B_{0}}=-\frac{1}{12} \omega \mu_{0} c_{0} \mathcal{N} \sum_{\epsilon \tau \nu} \varepsilon_{\epsilon \tau \nu} \Re\left|\left\langle\mu_{\epsilon} ; \mu_{\tau}, h_{B_{0}, \nu}^{\mathrm{Z}}\right\rangle\right|_{\omega, 0}, \\
& \frac{\phi^{\left(B_{0}\right)}}{l B_{0}}=-\frac{1}{12} \omega \mu_{0} c_{0} \mathcal{N} \sum_{\epsilon \tau \nu} \varepsilon_{\epsilon \tau \nu} \Im\left\langle\left\langle\mu_{\epsilon} ; \mu_{\tau}, h_{B_{0}, \nu}^{\mathrm{Z}}\right\rangle\right\rangle_{\omega, 0},
\end{aligned}
$$

where $\boldsymbol{\mu}=-e \sum_{i} \boldsymbol{r}_{i}$ is the electric dipole moment operator and $\boldsymbol{h}_{B_{0}}^{\mathrm{Z}}$ is the Hamiltonian for the Zeeman interaction with $\boldsymbol{B}_{0}$. In nonrelativistic (NR) electronic structure theory of closed-shell molecules, the latter corresponds to the orbital Zeeman $(\mathrm{OZ})$ interaction

$$
H_{B_{0}}^{\mathrm{OZ}}=-\boldsymbol{m} \cdot \boldsymbol{B}_{0}=\sum_{\nu} h_{B_{0}, v}^{\mathrm{OZ}} B_{0, v} ; \quad h_{B_{0}, v}^{\mathrm{OZ}}=\frac{e}{2 m_{e}} \sum_{i} \ell_{i O, v},
$$

where $\boldsymbol{m}$ is the magnetic dipole moment operator and $\ell_{i O}=-\imath \hbar\left[\left(\boldsymbol{r}_{i}-\boldsymbol{R}_{O}\right) \times \nabla_{i}\right]$ is the orbital angular momentum of electron $i$, at location $\boldsymbol{r}_{i}$, with respect to the gauge origin $\boldsymbol{R}_{O}$. The notation $\langle\langle A ; B, C\rangle\rangle_{\omega, 0}$ denotes third-order timedependent perturbation theory expressed in terms of a QRF involving dynamic (with frequency $\omega$ ) operators $A$ and $B$ and a static operator $C .{ }^{26}$ Standard perturbation theory fails when $\hbar \omega$ approaches the excitation energies of the system, leading to divergences of the response function (see, e.g., Ref. 6). In this work, we use the CPP approach by Norman et al., ${ }^{35,36}$ which includes relaxation of the excited states and allows for a smooth evaluation of the QRF over the entire frequency range, with results identical to those of the standard approach in nonabsorptive spectral regions and a well-behaving response also at resonance. The first MCD calculations using the CPP formalism were carried out by Solheim et al. ${ }^{37}$ The resonance linewidth is controlled by the parameter $\gamma$ that may be selected to empirically reproduce the experimental band shapes, which, in turn, are influenced by significant vibrational broadening not explicitly included in the present calculations. We note that molar $\eta^{\left(B_{0}\right)}$ and $\phi^{\left(B_{0}\right)}$ may be obtained from Eqs. (5) and (6) using $\mathcal{N}=n N_{A}$, where $n$ is the concentration of the molecules and $N_{A}$ is the Avogadro constant.

\section{B. Nuclear spin-induced circular dichroism}

In the NSCD experiment proposed here, the magnetization of a sample of spin-polarized nuclei is aligned with $\boldsymbol{k}$. This produces a magnetic field in the medium that is able to cause magneto-optic effects, even though no significant external magnetic field $\boldsymbol{B}_{0}$ is influencing the experiment. Various means of creating and controlling the nuclear magnetization in NMOS measurements have been discussed. ${ }^{9-12}$ These include transferring the magnetized sample from a separate polarization vessel to the optical apparatus, ${ }^{9,10}$ a method relying on relatively slow nuclear spin relaxation processes. Alternatively, the optical measurement can be placed transversally to the bore of a nuclear magnetic resonance spectrometer, enabling the creation and manipulation of the magnetization in the same volume. ${ }^{11,12}$
Consider now a sample of magnetized nuclei $K$ with the number density $\mathcal{N}_{K}=n_{K} N_{A}$, where $n_{K}$ is the molar concentration of the nuclei and the degree of nuclear spin polarization along $\boldsymbol{k}$ is equal to $P_{K}=\left\langle I_{K, Z}\right\rangle / I_{K}$, with $\left\langle I_{K, Z}\right\rangle$ the ensemble average of the spin component along $\hat{\boldsymbol{Z}}$ and $I_{K}$ the nuclear spin quantum number. The antisymmetric polarizability appearing in Eqs. (2) and (3) is now obtained from

$$
\left\langle\alpha_{X Y}^{\prime}\right\rangle=\frac{1}{6} P_{K} I_{K} \sum_{\epsilon \tau \nu} \varepsilon_{\epsilon \tau \nu} \alpha_{\epsilon \tau, \nu}^{\prime\left(I_{K}\right)},
$$

and the NSCD ellipticity and NSOR angle per unit sample length, spin polarization, and nuclear concentration can be written as

$$
\begin{aligned}
\eta_{K} & =\frac{\eta^{\left(I_{K}\right)}}{l P_{K} n_{K}} \\
& =-\frac{1}{12} \omega \mu_{0} c_{0} N_{A} I_{K} \sum_{\epsilon \tau \nu} \varepsilon_{\epsilon \tau \nu} \Re\left|\left\langle\mu_{\epsilon} ; \mu_{\tau}, h_{K, \nu}^{\mathrm{hf}}\right\rangle\right\rangle_{\omega, 0,} \\
V_{K} & =\frac{\phi^{\left(I_{K}\right)}}{l P_{K} n_{K}} \\
& =-\frac{1}{12} \omega \mu_{0} c_{0} N_{A} I_{K} \sum_{\epsilon \tau \nu} \varepsilon_{\epsilon \tau \nu} \Im\left\langle\left.\left\langle\mu_{\epsilon} ; \mu_{\tau}, h_{K, \nu}^{\mathrm{hf}}\right\rangle\right|_{\omega, 0} .\right.
\end{aligned}
$$

Here, the QRF notation involves the hyperfine interaction $\boldsymbol{h}_{K}^{\mathrm{hf}}$ that, in the NR theory for closed-shell systems, is the orbital hyperfine (paramagnetic nuclear spin-electron orbit, PSO) operator

$$
H_{K}^{\mathrm{PSO}}=\sum_{\nu} h_{K, v}^{\mathrm{PSO}} I_{K, v} ; \quad h_{K, v}^{\mathrm{PSO}}=\frac{e \hbar}{m_{e}} \frac{\mu_{0}}{4 \pi} \gamma_{K} \sum_{i} \frac{\ell_{i K, v}}{r_{i K}^{3}},
$$

where $\boldsymbol{\ell}_{i K}=-\imath \hbar\left[\left(\boldsymbol{r}_{i}-\boldsymbol{R}_{K}\right) \times \nabla_{i}\right]$ is the angular momentum of $i$ about the position $\boldsymbol{R}_{K}$ of nucleus $K$. It is seen that the NSCD and NSOR equations (9) and (10) are obtained from the expressions (5) and (6) of the corresponding observables for MCD and Faraday rotation, respectively, due to the external magnetic field, by exchanging the Zeeman interaction of the latter for the hyperfine interaction. In Eqs. (9) and (10), we have introduced the NSCD and NSOR constants $\eta_{K}$ and $V_{K}$ for nucleus $K$, respectively. A convenient unit for these quantities is $\mathrm{rad} /(\mathrm{M} \mathrm{cm})$.

\section{CALCULATIONS}

The calculation of NSCD [Eq. (9)] and NSOR [Eq. (10)] using the CPP approach was implemented into a development version of the DALTON program package. ${ }^{45}$ The efficient response equation solver introduced in Ref. 46 was used. We performed HF and DFT calculations of these properties for ethene, benzene, $\mathrm{pBQ}$, and ethanol molecules at fixed groundstate geometries in vacuo, thus a priori neglecting all rovibrational and solvent effects. The following geometries were used: ethene and benzene, the $r_{z}$ geometries of Refs. 47 and 48 , respectively; $\mathrm{pBQ}$ and ethanol, $r_{e}$ geometries optimized using DFT with the B3LYP functional ${ }^{49,50}$ and the aug-ccpVTZ basis set, ${ }^{51}$ on the GAUSSIAN 09 software. ${ }^{52}$ 
The basis-set requirements of the excited-state calculations (energy and transition moment from the ground state) were investigated using DALTON for ethene at the CAMB3LYP level ${ }^{56}$ using the correlation-consistent basis sets augcc-pVXZ, aug-cc-pCVXZ, as well as the doubly and triplyaugmented d/t-aug-cc-pVXZ $(X=\mathrm{D}, \mathrm{T}, \mathrm{Q}, 5,6)$ sets. $^{51,53}$ The lowest excitation energies and the corresponding oscillator strengths of these systems were investigated at the HF level as well as with various DFT functionals: PBE ${ }^{54} \mathrm{PBE} 0,{ }^{54}$ BLYP,${ }^{50,55}$ B3LYP ${ }^{49,50}$ CAM-B3LYP,${ }^{56}$ BHandHLYP,${ }^{50,57}$ as well as the following correlated ab initio coupled-cluster (CC) levels of presumably increasing accuracy: CC $2,{ }^{58} \mathrm{CCSD},{ }^{59}$ and CC $3 .{ }^{60}$ The purpose of using the $\mathrm{CC}$ methods was to assess the accuracy of HF and the various DFT functionals that were, in turn, used for NSCD and NSOR.

The basis-set requirements of NSCD and NSOR (both for ${ }^{1} \mathrm{H}$ and ${ }^{13} \mathrm{C}$ nuclei) were investigated for ethene using BHandHLYP at standard visible laser wavelengths, in the dispersive spectral range. This was not done in the transition region of the spectrum, as the results would have reflected the large dependence of the frequency of the transitions on the basis set. We also carefully checked that the applied numerical criteria for the wave function and response equation convergence, as well as the DFT grid, were sufficiently tight. The BLYP (with exact HF exchange admixture of 0\%), B3LYP (20\%), and BHandHLYP (50\%) series of generalized gradient/hybrid functionals, as well as $\mathrm{HF}$ and the range-separated CAM-B3LYP hybrid functional, were then used with the chosen d-aug-cc-pCVTZ basis ${ }^{53}$ (with highexponent core-valence correlation functions) for the production calculations in the region of selected low-lying transitions of the target systems. For comparison, MCD calculations using the method of Solheim et al. ${ }^{37}$ were also performed for the same transitions.

We used the empirical linewidth factor $\gamma$ equal to $1000 \mathrm{~cm}^{-1}$ (0.00456 a.u.) and the step between successive frequencies in the transition region equal to 0.0025 a.u. The choice of $\gamma$ affects the calculated spectra, a larger value pro- duces broader spectral features with lower peak intensity. In the present kind of pure electronic structure calculations where various factors (such as vibronic couplings) affecting the experimental linewidths are not considered, the value of $\gamma$ can be empirically adjusted to approximately to match the observed spectra. So far there exist no experiments for NSCD or NSOR in the absorptive spectral region, and we rely in our selection of $\gamma$ on the previous application ${ }^{37}$ of the CPP theory for MCD in systems of composition comparable to the present ones.

The NMOS properties corresponding to all the experimentally non-equivalent nuclei of the molecules were computed. In the case of ethanol, the results were averaged over all the protons in, on the one hand, $-\mathrm{CH}_{3}$ and, on the other hand, $-\mathrm{CH}_{2}-$ group.

\section{RESULTS AND DISCUSSION}

\section{A. Excitation energies and oscillator strengths}

\section{Basis-set convergence}

Figure 1 illustrates the basis-set convergence of the calculated two lowest electric dipole-allowed excitation energies in ethene. Results with the various augmented correlationconsistent basis-set families are shown as obtained with the CAM-B3LYP functional. The corresponding convergence pattern of the electric-dipole transition moments is displayed in Figure S1 of the supplementary material. ${ }^{63}$ The numerical data corresponding to the figures are listed in Table S1 of the supplementary material. ${ }^{63}$

A point to note is that the electric-dipole transition moments and oscillator strengths only have an indirect relevance for the NMOS observables, which are the primary objective of this work. Because NSCD and NSOR (similar to MCD) involve, apart from the dynamic electric dipole interaction, also a static magnetic interaction operator, the relative intensities of the magneto-optic signals do not directly correspond to calculated dipolar oscillator strengths.
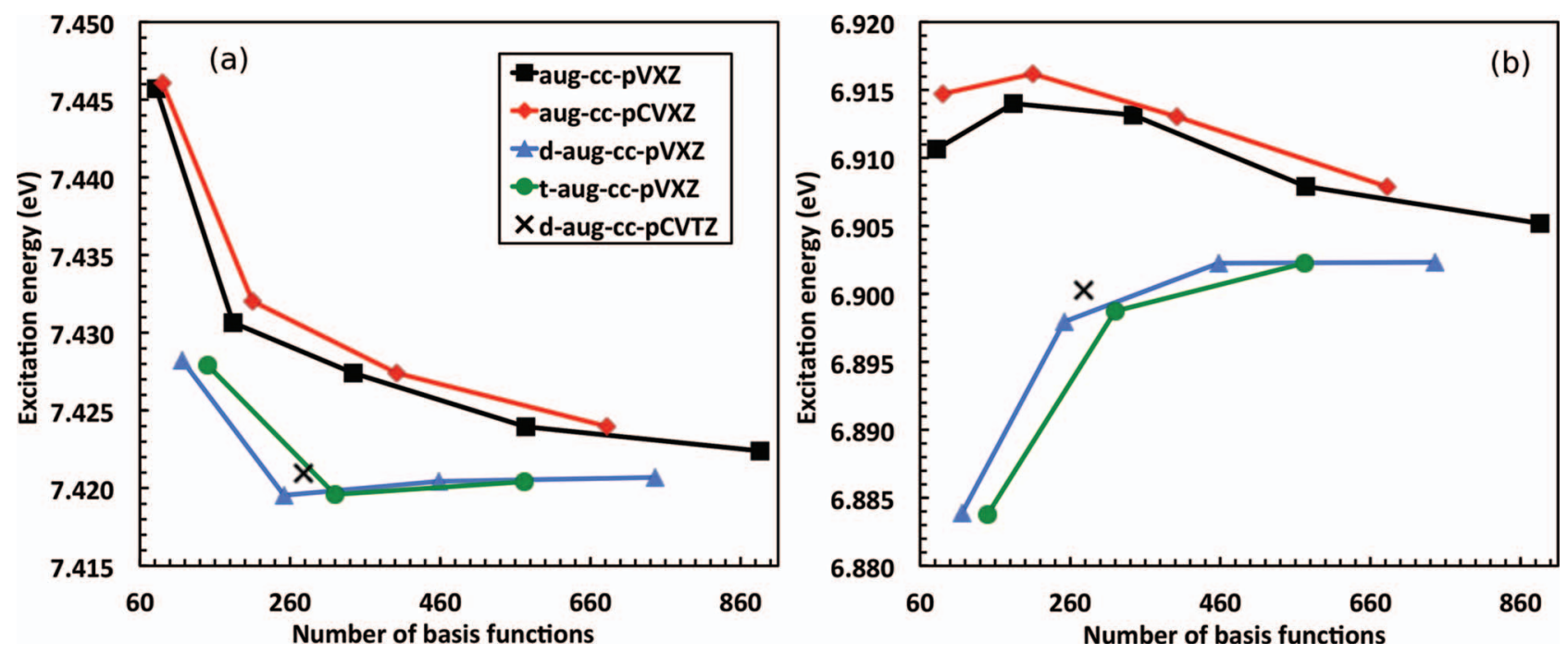

FIG. 1. Basis-set convergence of the calculated, low-lying electric dipole-allowed excitation energies of ethene $\left(\mathrm{C}_{2} \mathrm{H}_{4}\right)$ at the $\mathrm{CAM}-\mathrm{B} 3 \mathrm{LYP}$ level. Results at different correlation-consistent basis-set levels. The basis set selected for the production calculations of NSCD and NSOR is marked with a cross. (a) Excitation energy of the $\mathrm{X}^{1} \mathrm{~A}_{g} \rightarrow 1^{1} \mathrm{~B}_{3 u}$ transition. (b) Excitation energy of the $\mathrm{X}^{1} \mathrm{~A}_{g} \rightarrow 1^{1} \mathrm{~B}_{1 u}$ transition. 
The results in Figures 1 and $\mathrm{S} 1$ (supplementary material ${ }^{63}$ ) indicate the convergence of all the basis set series towards a common basis-set limit. The singly augmented sets (aug-cc-pVXZ) are expectedly the slowest in this approach, whereas no significant difference is obtained between the doubly (d-aug) and triply (t-aug) augmented sets. The singly augmented sets were used in both the standard version (aug-cc-pVXZ) and the aug-cc-pCVXZ series, in which tight core-valence correlation functions have been added. Whereas the latter offer no advantage for the valence excitation energies and oscillator strengths, they are generally found important for hyperfine properties. The figures show with a cross the results obtained with the d-aug-cc-pCVTZ basis selected presently for the production calculations of the magneto-optic properties. This basis is reasonable close to the basis-set limit.

\section{Comparison of methods}

Figure 2 illustrates the excitation energies for transitions from the ground state to two lowest dipole-allowed excited states in ethene, calculated with the t-aug-cc-pVDZ basis set. Different (HF, CC2, CCSD, CC3, and DFT) methods were used. For the latter, a selection of functionals was chosen. The corresponding oscillator strengths are given in Figure S2 of the supplementary material. ${ }^{63}$ The numerical data are listed in Table $\mathrm{S} 2$ of the supplementary material. ${ }^{63}$

The present correlated $a b$ initio estimates (CC2, CCSD, CC3) are in good agreement with Refs. 61 and 62 for the excitation energies of both the $\mathrm{B}_{3 u}$ and $\mathrm{B}_{1 u}$ states. The various DFT functionals give a systematically increasing deviation (decreasing excitation energies) from these reference values as the exact exchange parameter diminishes, towards the pure GGA functional (BLYP). BHandHLYP (50\% of exact exchange) and the range-separated CAM-B3LYP functional fare best in this context. These two functionals also display a reasonable performance for the oscillator strengths, as referenced to the $\mathrm{CC} 3$ and CCSD results.

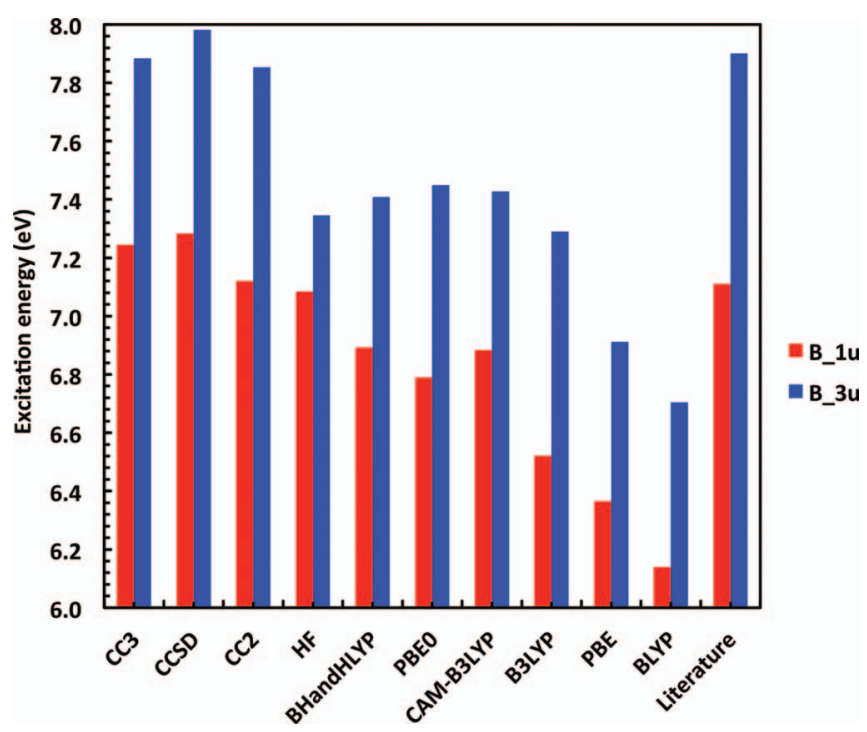

FIG. 2. Calculated vertical excitation energies (in eV) in ethene $\left(\mathrm{C}_{2} \mathrm{H}_{4}\right)$ using different electron correlation methods and the t-aug-cc-pVDZ basis set.
Figure S3 and Tables S3-S5 of the supplementary material ${ }^{63}$ ) contain the excitation energies and oscillator strengths for the three lowest dipole-allowed states in benzene, one state in $\mathrm{pBQ}$, and four states in ethanol. Literature values also shown for benzene $e^{64,65}$ and $\mathrm{pBQ} .^{66-68}$ Common to all these cases is that we went only up to CCSD level in $a b$ initio calculations of both the excitation energies and oscillator strengths, as we reckoned that the more accurate CC3 method would have proved computationally too expensive. Similarly, the comparison with the available literature values is impaired by the different molecular geometries used between calculations, as well as the approximations made in the analysis of experimental data. Our present purpose is to assess the accuracy of the various DFT methods for these excited states, and we pick the CCSD data as the principal point of comparison. More thorough investigations of the performance of various methods in calculating excitation energies exist in the literature, and we quote Ref. 68 for a recent example. In the present calibration, we note that the BHandHLYP and CAM-B3LYP functionals provide again the best match among the DFT functionals with the CCSD excitation energies for benzene and ethanol, and somewhat less successfully in the case of $\mathrm{pBQ}$. The quantitative agreement is generally worse for the oscillator strengths, however the two mentioned functionals give overall the best results among the present single-determinantal methods.

\section{B. Nuclear magneto-optic properties}

\section{Basis-set convergence}

A note is in place concerning the sign conventions used for optical rotation. According to the chemical convention, ${ }^{1}$ the formulae presented in this paper lead for closed-shell molecules to a negative rotation angle, both in normal Faraday rotation due to the external field and in NSOR for nuclei with a positive $\gamma_{K}$. A negative angle corresponds to the rotation of the plane of polarization to the direction of the positive electric current in a solenoid that generates the external field. The convention normally used in the literature reporting Verdet constants is exactly the opposite (see, e.g., Ref. 69) and a positive Verdet constant is reported under the same circumstances. We follow the chemical convention in this paper.

We first verified the consistency of the new CPP implementation with the standard response theory method for NSOR, which was used in earlier reports of this property. ${ }^{6,10,15,16}$ Calculated NSOR results at off-resonant wavelengths for ethene, listed in Table S6 of the supplementary material, ${ }^{63}$ confirm that far from the transition region the damped response theory results indeed are in a very good agreement with the results of the standard approach.

Figure 3 and Table S7 of the supplementary material ${ }^{63}$ contain the data for the basis-set convergence of the calculated NSCD ellipticity and NSOR angle for both the ${ }^{13} \mathrm{C}$ and ${ }^{1} \mathrm{H}$ nuclei in ethene, obtained at the BHandHLYP level.

Both the NSCD ellipticity and NSOR angle increase in absolute value upon shortening the wavelength, towards the first optical transition that appears at $180 \mathrm{~nm}$ at the BHandHLYP/d-aug-cc-pCVTZ level of theory used in Figures 3(a) and 3(b). Panels (c)-(f) illustrate the difference 

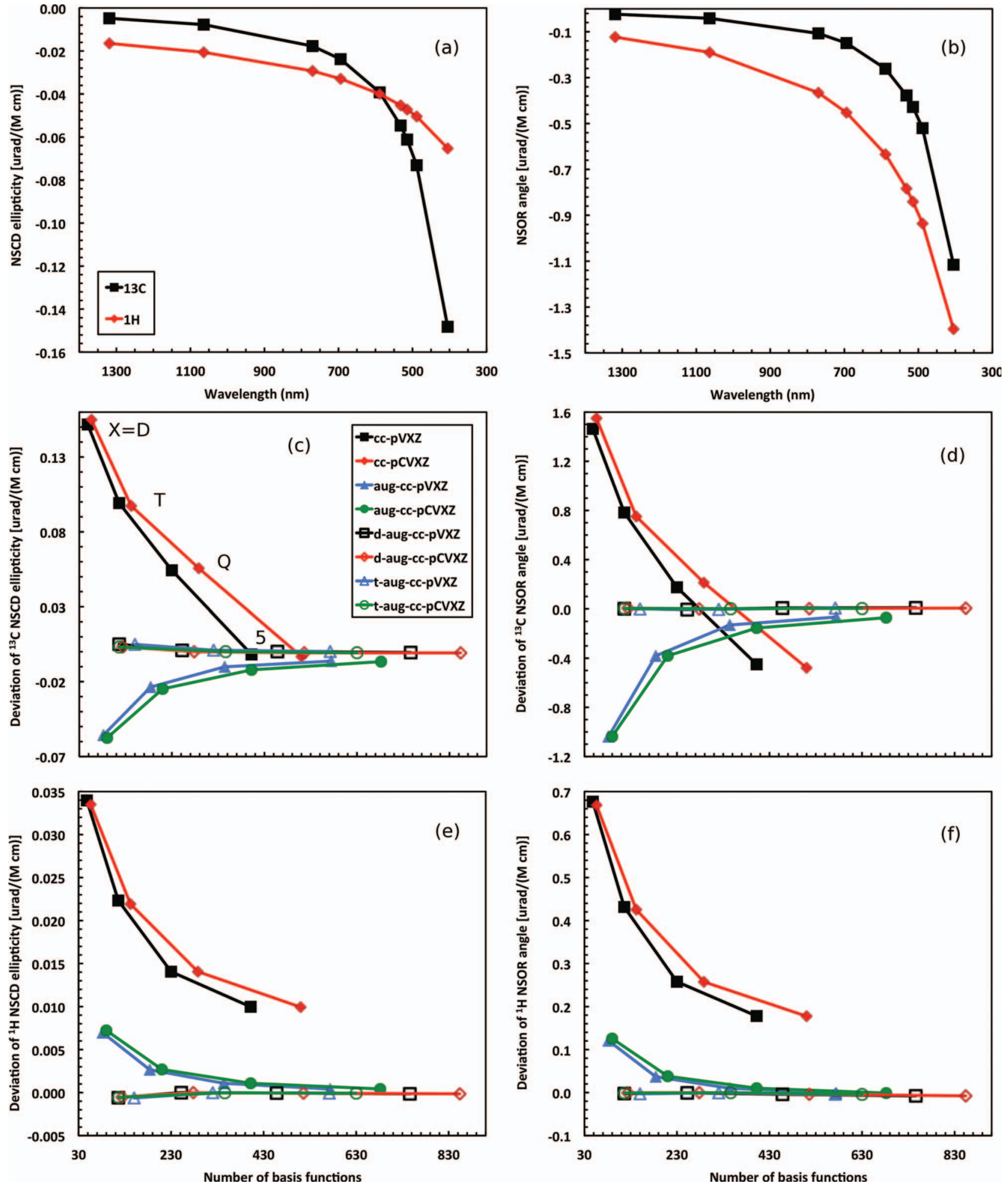

FIG. 3. Basis-set dependence of the calculated NSCD and NSOR constants, $\eta_{K}$ and $V_{K}$, respectively, for ethane $\left(\mathrm{C}_{2} \mathrm{H}_{4}\right)$ in units of $\mu \mathrm{rad} /(\mathrm{M} \mathrm{cm})$. The DFT functional BHandHLYP was used. Results for both $K={ }^{1} \mathrm{H}$ and ${ }^{13} \mathrm{C}$. (a) NSCD and (b) NSOR results with d-aug-cc-pCVTZ as functions of $\lambda$. Also shown are the deviations from d-aug-cc-pCVTZ results at $\lambda=405.0 \mathrm{~nm}$ with different basis sets, as a function of the number of basis functions: (c) ${ }^{13} \mathrm{CSCD}$ and (d) ${ }^{13}$ CSOR, (e) ${ }^{1} \mathrm{HSCD}$ and (f) ${ }^{1} \mathrm{HSOR}$.

of results obtained with the various basis sets as compared to the d-aug-cc-pCVTZ set, at $\lambda=405 \mathrm{~nm}$. It is seen that the unaugmented cc-pVXZ and cc-pCVXZ sets do not perform satisfactorily as the results not only converge quite slowly with the size of the basis set, but they even appear to converge to erroneous limiting values. In contrast, the singly, doubly, and triply augmented sets converge to a common limit, and hardly any difference can be seen between the behavior of the two latter series. The changes of the results due to supplementing the basis with tight core correlating functions in the 



FIG. 4. Calculated magnetic response properties of ethene with various DFT functionals and with Hartree-Fock (HF): (a) MCD and (b) Faraday optical rotation [in $\mathrm{mrad} /(\mathrm{T} \mathrm{M} \mathrm{cm})]$ due to the external magnetic field, and the following nuclear magneto-optic quantities [in $\mu \mathrm{rad} /(\mathrm{M} \mathrm{cm})]:(\mathrm{c}){ }^{13} \mathrm{CSCD},(\mathrm{d}){ }^{13} \mathrm{CSOR}$, (e) ${ }^{1}$ HSCD, and (f) ${ }^{1}$ HSOR. d-aug-cc-pCVTZ basis set was used.

cc-pCVXZ series as opposed to the cc-pVXZ sets, are relatively small. We select the d-aug-cc-pCVTZ set as a computationally manageable, fairly accurate set for the remaining calculations further in this paper. At the DFT level, the truncation error implied by this basis set is about $1 \%$ for the presently investigated properties.

\section{Results for ethene, benzene, and para-benzoquinone}

Figure 4 shows the calculated magneto-optic properties in ethene as functions of the wavelength $\lambda$ around the two lowest dipole-allowed excitations using the HF method, as well as 
DFT, employing functionals with decreasing exact exchange admixture: BHandHLYP, B3LYP, and BLYP. In addition, results with the range-separated CAM-B3LYP functional are also shown. The corresponding numerical data are collected in Table S8 of the supplementary material. ${ }^{63}$

The results show a consistent shift of the spectral features towards longer wavelengths when moving from HF to the DFT functionals with a decreasing exact exchange admixture, in accordance with the behaviour of the excitation energies (Table S2 of the supplementary material). ${ }^{63}$ Based on the performance of the various methods in the calculation of excitation energies and oscillator strengths, we focus mainly on the BHandHLYP and CAM-B3LYP data, which are mutually remarkably similar not only for ethene but also for the other presently investigated molecules (vide infra).

The calculated MCD spectra of ethene in the rigid molecule limit were discussed and compared with the experiment in Ref. 28. The present BHandHLYP and CAM-B3LYP calculations [Figure 4(a)] produce two negative-ellipticity absorption bands centred at the locations of the two excitations, about 180 and $167 \mathrm{~nm}$ (quoting BHandHLYP/CAM-B3LYP data), corresponding to ${ }^{1} \mathrm{~B}_{1 u}$ (transition dipole directed normal to the molecular plane) and ${ }^{1} \mathrm{~B}_{3 u}$ (along the direction of the $\mathrm{CC}$ bond) excited states, respectively. The corresponding Faraday optical rotation spectrum [Figure 4(b)] consists of two overlapping derivative bands. The MCD and Faraday rotation results with the standard B3LYP and BLYP functionals have additional features in the spectral regions towards smaller wavelengths, on account of the proximity of further optical excitations at these levels of theory.

The carbon-13 NSCD signal in ethene [Figure 4(c)] also shows two peaks with the absorption lineshape, but in contrast to MCD, the spectrum consists of a negative peak at $180 \mathrm{~nm}$ and a positive peak at $167 \mathrm{~nm}$. The proton NSCD [Figure 4(e)] features similarly a combination of two antiphase absorption signals, but for this nucleus the ${ }^{1} \mathrm{~B}_{1 u}$ excitation is characterized by a positive peak. The NMOS observables depend on the gyromagnetic ratio $\gamma_{K}$ of the nucleus in question [Eqs. (9)-(11)]. As the ${ }^{13} \mathrm{C}$ and ${ }^{1} \mathrm{H}$ nuclei have $\gamma_{K}$ of the same (positive) sign, the difference in the signs of the corresponding NSCD signals is not due to the properties of these nuclei but is a feature of the electronic structure. While the two absorption peaks in the ${ }^{13} \mathrm{C}$ spectrum are almost of equal intensity, the protons cause a much larger signal at the ${ }^{1} \mathrm{~B}_{3 u}$ excitation. It should be kept in mind that the detailed lineshape does depend significantly on the choice of the computational method, with particularly the HF method giving rather different results. Overall the ${ }^{13} \mathrm{CSCD}$ signals are roughly 50 times more intense in ethene than the ${ }^{1} \mathrm{H}$ signals, possibly due to the larger electronic density at the carbon sites, which by far overcompensates the fact that the magnetic moment of proton is four times larger than that of ${ }^{13} \mathrm{C}$.

The ${ }^{13} \mathrm{C}$ and ${ }^{1} \mathrm{H}$ NSOR spectra of ethene [Figures 4(d) and 4(f), respectively] consist of two overlapping lines with the derivative lineshape and, similarly as in the corresponding NSCD spectra, the signals of these two nuclei have opposite signs. The present damped response theory calculations verify qualitatively the dramatic amplification of the NSOR signals around optical transitions, predicted earlier using conven- tional response theory. ${ }^{6,24}$ Hence, future NSCD experimental set-ups could use both the ellipticity and optical rotation as means of detecting nuclear site-specific magneto-optic signals. The same enhancement factor of 50 is valid also for the ${ }^{13} \mathrm{CSOR}$ signals as compared to those of proton, like in the NSCD case.

Figure 5 shows the results of the magneto-optic calculations for benzene in the region of the three lowest optical transitions of relevance, to the ${ }^{1} \mathrm{~B}_{1 u}$ state with the transition dipole perpendicular to the molecular plane at around 180-182 nm (CAM-B3LYP and BHandHLYP wavelengths quoted) and two in-plane excitations to ${ }^{1} \mathrm{E}$ states at circa 177 and 171-172 $\mathrm{nm}$. The corresponding numerical data are listed in Table S9 of the supplementary material. ${ }^{63}$ The data for para-benzoquinone are given in Fig. 6 (for ${ }^{13} \mathrm{C}$ and $\left.{ }^{1} \mathrm{H}\right)$, Fig. S4 $\left({ }^{17} \mathrm{O}\right)$ and Table $\mathrm{S} 10$ of the supplementary material, ${ }^{63}$ and they involve the transition to ${ }^{1} \mathrm{~B}_{1 u}$ excited state at 227-233 nm (BHandHLYP and CAM-B3LYP levels). In both cases, we choose to display only the BHandHLYP and CAM-B3LYP data in the figures. The results obtained with the other functionals employed are included in the tables.

In the case of benzene, the MCD spectrum consists of one strong negative-ellipticity and two weaker, positive absorption features corresponding to the $\mathrm{B}_{1 u}$ and two $\mathrm{E}$ states, respectively. As before for ethene, the NSCD spectra do not stand in one-to-one correspondence with MCD; while the ${ }^{13}$ CSCD signal of benzene follows the sign pattern of MCD, the proton spectrum shows the opposite signature. The intensity of the lower of the two E-type bands is equally large with the $\mathrm{B}_{1 u}$ band both in ${ }^{13} \mathrm{C}$ and ${ }^{1} \mathrm{HSCD}$, in contrast to MCD. The NSOR signals display consistently the derivative patterns corresponding to the features in the respective $\mathrm{CD}$ spectra. The comparison of the magnitudes of the ${ }^{1} \mathrm{H}$ and ${ }^{13} \mathrm{C}$ signals indicates that the large amplification factor observed in $\mathrm{C}_{2} \mathrm{H}_{4}$ for carbon as compared to hydrogen is not a universal feature: in benzene the carbon signals are only roughly twice as intense as those of proton.

We investigated only one transition for $p \mathrm{BQ}$ in the 200$300 \mathrm{~nm}$ region, and for this molecule MCD shows a positive absorption peak, whereas all the ${ }^{13} \mathrm{C},{ }^{17} \mathrm{O}$, and ${ }^{1} \mathrm{H}$ NSCD spectra show a single negative peak, matched by a derivative lineshape in the respective OR signals. Again, the intensities of the ${ }^{13} \mathrm{C}$ and ${ }^{1} \mathrm{H}$ signals obey roughly the $2: 1$ pattern, akin to benzene. The ${ }^{17} \mathrm{O}$ spectrum (Figure $\mathrm{S} 4$ in the supplementary material ${ }^{63}$ ) is similar in lineshape to that of ${ }^{13} \mathrm{C}$ but more intense by two orders of magnitude. Despite the fact these two isotopes have oppositely signed gyromagnetic ratios, their NSCD and NSOR signals, which are directly proportional to the nuclear magnetic moment, have the same phase in $\mathrm{pBQ}$. Hence, the purely electronic NSCD/NSOR response corresponding to these two nuclei is different in both magnitude and sign.

From the comparison of MCD and NSCD signals in $\mathrm{C}_{2} \mathrm{H}_{4}, \mathrm{C}_{6} \mathrm{H}_{6}$, and $p \mathrm{BQ}$ it may be concluded that each system has a unique pattern of the signs and intensities of the spectral features, with the NSCD signals not always following those of MCD. The qualitative interpretation of the NSCD spectra will require further conceptual work. 



FIG. 5. Calculated magnetic response properties of benzene with BHandHLYP and CAM-B3LYP DFT functionals: (a) MCD and (b) Faraday optical rotation [in $\mathrm{mrad} /(\mathrm{T} \mathrm{M} \mathrm{cm})$ ] due to the external magnetic field, and the following nuclear magneto-optic quantities [in $\mu \mathrm{rad} /(\mathrm{M} \mathrm{cm})]$ : $(\mathrm{c}){ }^{13} \mathrm{CSCD}$, (d) ${ }^{13} \mathrm{CSOR}$, (e) ${ }^{1}$ HSCD, and (f) ${ }^{1}$ HSOR. d-aug-cc-pCVTZ basis set was used.

\section{Nuclear spin CD for non-equivalent nuclear sites: Ethanol}

We chose to use ethanol to investigate the nuclear sitespecificity of the NSCD spectroscopic signals. The two nonequivalent carbons $\left(\mathrm{C} 1\right.$ and $\mathrm{C} 2$ in the $-\mathrm{CH}_{3}$ and $-\mathrm{CH}_{2}-$ moieties, respectively) and the three non-equivalent protons $\left(-\mathrm{CH}_{3},-\mathrm{CH}_{2}-\right.$, and $-\mathrm{OH}$ groups, averaged over the experimentally equivalent nuclei in the first two cases) are expected to reflect the chemical environment of the nucleus in NSCD, similar to what was predicted (also for ethanol) in the case of NSOR signals in Ref. 6. We display the 



FIG. 6. (a)-(f) As Fig. 5 but for para-benzoquinone.

calculated magneto-optic spectra for ethanol using different computational methods (HF and the present DFT functionals) in Figures S5 of the supplementary material ${ }^{63}$ (MCD/OR and ${ }^{17} \mathrm{OSCD} /{ }^{17} \mathrm{OSOR}$ ), and specifically for ${ }^{13} \mathrm{C}$ and ${ }^{1} \mathrm{H}$ nuclei in Figure 7 at the BHandHLYP level. The HF and other DFT results for the latter nuclei are illustrated in Figures S6 and $\mathrm{S} 7$ of the supplementary material. ${ }^{63}$ The numerical data are tabulated in Tables S11 and S12 of the supplementary material. $^{63}$

The calculations of ethanol were carried out for the wavelength range around the four lowest dipole-allowed excitations, at 150,156 , and $178 \mathrm{~nm}$ to ${ }^{1} \mathrm{~A}^{\prime \prime}$ states and $150 \mathrm{~nm}$ to ${ }^{1} \mathrm{~A}^{\prime}$ state, wavelengths quoted from the BHandHLYP results. Investigation of the ${ }^{13} \mathrm{C}$ and ${ }^{1} \mathrm{H}$ NSCD signals [Figures 7(a) 

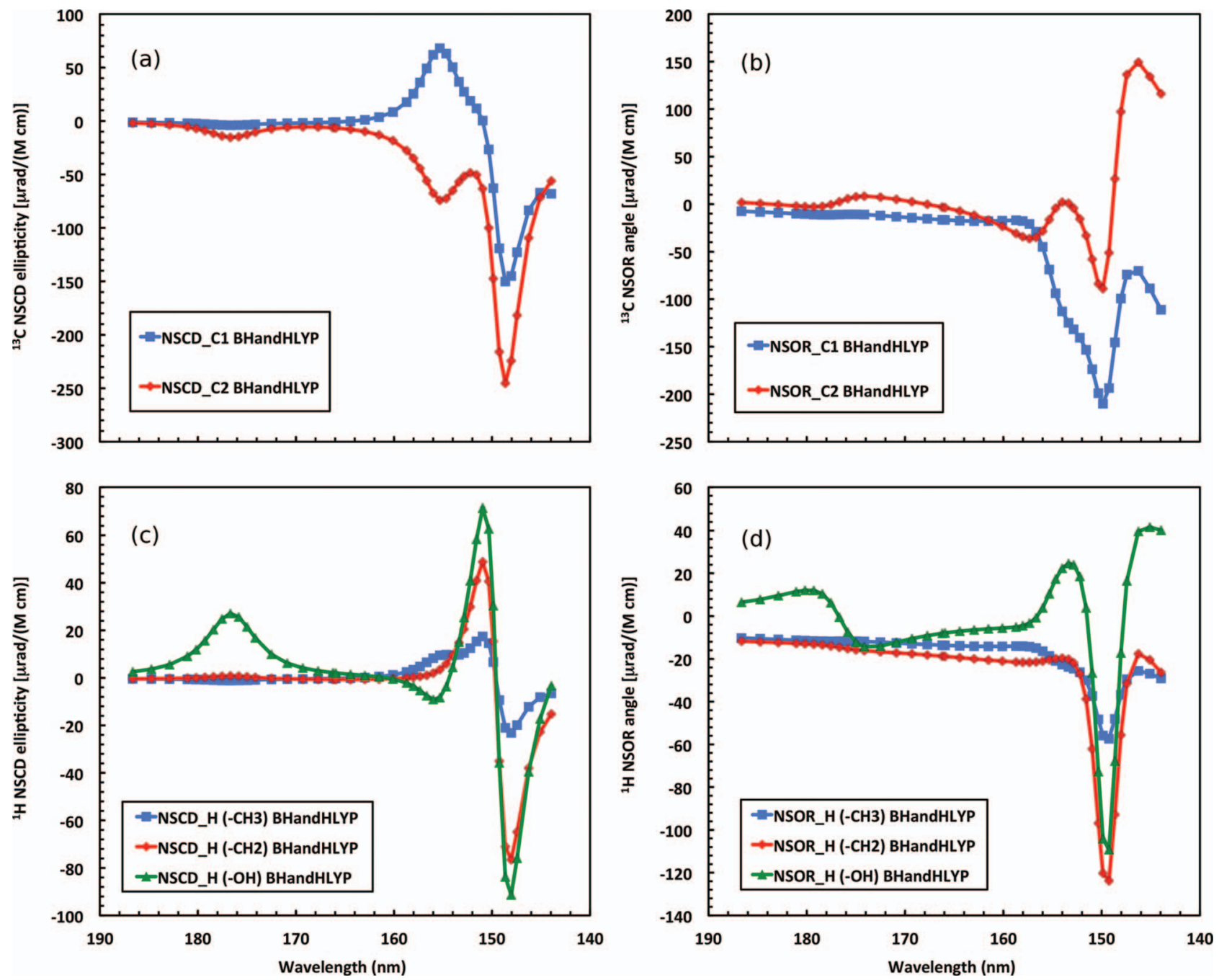

FIG. 7. Calculated (BHandHLYP/d-aug-cc-pCVTZ) nuclear magneto-optic properties in ethanol highlighting the differences between the signals of the unequivalent nuclei. (a) ${ }^{13} \mathrm{CSCD}$, (b) ${ }^{13} \mathrm{CSOR}$, (c) ${ }^{1} \mathrm{HSCD}$, and (d) ${ }^{1} \mathrm{HSOR}$.

and $7(\mathrm{c})$, respectively] reveals that the lowest-energy $\mathrm{X}^{1} \mathrm{~A}^{\prime}$ $\rightarrow 1^{1} \mathrm{~A}^{\prime \prime}$ transition is associated with a vanishing NSCD response in both the ${ }^{13} \mathrm{C}$ signal of the $-\mathrm{CH}_{3}$ group $(\mathrm{C} 1)$ and the ${ }^{1} \mathrm{H}$ spectra from both the $-\mathrm{CH}_{3}$ and $-\mathrm{CH}_{2}-$ groups. In contrast, $\mathrm{C} 2$ (the $-\mathrm{CH}_{2}-$ group) and the ${ }^{1} \mathrm{H}$ spectra corresponding to the $-\mathrm{OH}$ group show a clear response of these nuclei to this transition. This can be also noted form the corresponding ${ }^{17} \mathrm{O}$ signal [Figure S5(c) of the supplementary material]. ${ }^{63}$ Precisely the same nuclei respond in the corresponding NSOR signals in Figures 7(b) and 7(d) [Figure S5(d) of the supplementary material ${ }^{63}$ in the case of $\left.{ }^{17} \mathrm{O}\right]$. Consequently, both the NSCD observables and NSOR, calculated here using a methodology that is able to cope with frequencies in the immediate vicinity of the transitions, confirm the earlier findings ${ }^{6}$ concerning the NSOR of ethanol using standard response theory. NMOS observables allow nuclear site-specific magneto-optical spectroscopy. The $\mathrm{O}^{1} \mathrm{H}$ and ${ }^{13} \mathrm{CH}_{2}$ NSCD signals are found to be of roughly similar intensity at this transition.

The transition to the second ${ }^{1} \mathrm{~A}^{\prime \prime}$ state at $156 \mathrm{~nm}$ (BHandHLYP) gives further confirmation to this observation. Signals of opposite phases are found at this transition for $\mathrm{C} 1$ (positive) and $\mathrm{C} 2$ (negative), as well as for $-\mathrm{C}^{1} \mathrm{H}_{3}$ (positive) and $-\mathrm{O}^{1} \mathrm{H}$ (negative), whereas the $-\mathrm{CH}_{2}-$ group protons are unresponsive at $156 \mathrm{~nm}$. Finally, the spectra at around $150 \mathrm{~nm}$ result from transitions to both ${ }^{1} \mathrm{~A}^{\prime}$ and ${ }^{1} \mathrm{~A}^{\prime \prime}$ states, with the former placed at a slightly larger wavelength. The $\mathrm{X}^{1} \mathrm{~A}^{\prime} \rightarrow 1^{1} \mathrm{~A}^{\prime}$ transition results in a weak and strong positive signal for $\mathrm{C} 2$ and all the protons, respectively, particularly in the $-\mathrm{OH}$ and $-\mathrm{CH}_{2}-$ moieties. The $\mathrm{X}^{1} \mathrm{~A}^{\prime} \rightarrow 3^{1} \mathrm{~A}^{\prime \prime}$ transition yields consistently a negative feature in the NSCD spectra for all carbons and protons.

\section{CONCLUSIONS}

A novel form of nuclear magneto-optic spectroscopy, the nuclear spin-induced circular dichroism, which arises from the differential absorption of left- and right-circularly polarized light on account of the magnetic field created by the magnetization of individual nuclei in molecules, is proposed in this paper. In analogy to MCD, which is due to the externally applied magnetic field, NSCD can be experimentally detected from ellipticity induced to the plane-polarized light at wavelength regions close to the optical transitions of the molecule. 
Comparison with MCD on the one hand and Faraday optical rotation (due to an external magnetic field) on the other hand suggests that NSCD might yield an even more insightful direction for the ongoing experimental NMOS efforts than the already observed phenomenon of nuclear spin optical rotation. Strong signals from both phenomena are predicted in the absorptive spectral regions, and we propose experiments with a matching pair of tunable laser source and a medium consisting of, e.g., dye molecules with strong absorption band in the visible range.

We have formulated a third-order time-dependent perturbation theory expression for the NSCD ellipticity, expressed as the real part of the derivative of the molecular dynamic polarizability tensor with respect to the nuclear magnetic moment. A first-principles computation method for NSCD has been implemented to the DALTON quantum-chemical package, involving a variant of damped response theory, which enables calculations at frequencies in the immediate vicinity of the transition, as parameterized by an empirical linewidth factor.

Calculations of NSCD have been presented for lowlying transitions in a series of small organic molecules using the Hartree-Fock and DFT methods, demonstrating that the NSCD signals are of observable intensity and they yield a resolution of the varying chemical environments of the nuclei: a different sensitivity of the magneto-optic response results for magnetization of non-equivalent nuclei is observed. This optical chemical shift, analogous to the corresponding phenomenon in NSOR, paves way for high-resolution NMOS using the NSCD effect. The present damped response theory calculations also qualitatively verify earlier predictions of strongly enhanced NSOR signals when the photon energies approach the optical transitions.

All the present calculations of NSCD have been based on the responses of a single molecule to the optical and nuclear magnetic fields. The formulation of bulk magneto-optic properties has essentially been carried out by multiplying the calculated single-molecule properties by the number density of molecules in a medium. Previous research on NSOR ${ }^{14,16}$ suggests that the macroscopic nuclear magnetic polarization of the medium will give an additional, MCD-like, and nonnucleus-specific contribution to the differential absorption observed in NSCD experiments. Furthermore, modification of the local optical field will also occur in a medium. ${ }^{16}$ These additions to the basic NSCD theory will be topics for further research.

\section{ACKNOWLEDGMENTS}

The authors acknowledge discussions with Dr. Geert Rikken (Toulouse) who first suggested this topic to us. The work of J.V. was supported by the Academy of Finland, the University of Oulu, the Tauno Tönning Foundation, and Societas Scientiarum Fennica. S.C. acknowledges support from the Italian Ministero dell'Istruzione, dell'Università e della Ricerca within the PRIN2009 funding scheme [Grant No. 2009C28YBF_001, Modelli teorici per processi di fotoassorbimento e fotoemissione] and from the University of Trieste [grant CHIM02-Ricerca]. Computational resources were par- tially provided by CSC-IT Center for Science (Espoo, Finland) and the Finnish Grid Initiative project.

${ }^{1}$ L. D. Barron, Molecular Light Scattering and Optical Activity (Cambridge University Press, 1982).

${ }^{2}$ A. D. Buckingham and P. J. Stephens, Annu. Rev. Phys. Chem. 17, 399 (1966).

${ }^{3}$ M. Faraday, Philos. Trans. R. Soc. London 136, 1 (1846); Philos. Mag. 28, 294 (1846).

${ }^{4}$ P. J. Stephens, J. Chem. Phys. 52, 3489 (1970).

${ }^{5}$ A. Cotton and H. Mouton, C. R. Hebd. Séances Acad. Sci. 141, 317 (1905); 141, 349 (1905); A. Cotton, Rapp. Cons. Phys. Solvay 418 (1932).

${ }^{6}$ S. Ikäläinen, M. V. Romalis, P. Lantto, and J. Vaara, Phys. Rev. Lett. 105, 153001 (2010).

${ }^{7}$ T. Kjærgaard, S. Coriani, and K. Ruud, WIREs Comput. Mol. Sci. 2, 443 (2012), and references therein.

${ }^{8}$ C. Rizzo, A. Rizzo, and D. M. Bishop, Int. Rev. Phys. Chem. 16, 81 (1997), and references therein.

${ }^{9}$ I. M. Savukov, S.-K. Lee, and M. V. Romalis, Nature (London) 442, 1021 (2006).

${ }^{10}$ J. Shi, S. Ikäläinen, J. Vaara, and M. V. Romalis, J. Phys. Chem. Lett. 4, 437 (2013).

${ }^{11}$ D. Pagliero, W. Dong, D. Sakellariou, and C. A. Meriles, J. Chem. Phys. 133, 154505 (2010)

${ }^{12}$ D. Pagliero and C. A. Meriles, Proc. Natl. Acad. Sci. U.S.A. 108, 19510 (2011).

${ }^{13}$ I. M. Savukov, H.-Y. Chen, T. Karaulanov, and C. Hilty, J. Magn. Reson. 232, 31 (2013)

${ }^{14}$ G.-H. Yao, M. He, D.-M. Chen, T.-J. He, and F.-C. Liu, Chem. Phys. 387, 39 (2011).

${ }^{15}$ S. Ikäläinen, P. Lantto, and J. Vaara, J. Chem. Theory Comput. 8, 91 (2012).

${ }^{16}$ T. S. Pennanen, S. Ikäläinen, P. Lantto, and J. Vaara, J. Chem. Phys. 136, 184502 (2012).

${ }^{17}$ T. Lu, M. He, D. Chen, T. He, and F.-C. Liu, Chem. Phys. Lett. 479, 14 (2009).

${ }^{18}$ G.-H. Yao, M. He, D.-M. Chen, T.-J. He, and F.-C. Liu, ChemPhysChem 13, 1325 (2012).

${ }^{19}$ L.-J. Fu and J. Vaara, J. Chem. Phys. 138, 204110 (2013).

${ }^{20}$ L.-J. Fu, A. Rizzo, and J. Vaara, J. Chem. Phys. 139, 181102 (2013).

${ }^{21}$ L.-J. Fu and J. Vaara, J. Chem. Phys. 140, 024103 (2014).

${ }^{22}$ J. T. Arnold, S. S. Dharmatti, and M. E. Packard, J. Chem. Phys. 19, 507 (1951).

${ }^{23}$ A. D. Buckingham and L. C. Parlett, Mol. Phys. 91, 805 (1997).

${ }^{24}$ R. H. Romero and J. Vaara, Chem. Phys. Lett. 400, 226 (2004).

${ }^{25}$ S. Ikäläinen, P. Lantto, P. Manninen, and J. Vaara, J. Chem. Phys. 129, 124102 (2008).

${ }^{26}$ J. Olsen and P. Jørgensen, J. Chem. Phys. 82, 3235 (1985).

${ }^{27}$ P. Štěpánek, M. Straka, V. Andrushchenko, and P. Bouř, J. Chem. Phys. 138, 151103 (2013).

${ }^{28}$ S. Coriani, P. Jørgensen, A. Rizzo, K. Ruud, and J. Olsen, Chem. Phys. Lett. 300, 61 (1999); H. Solheim, L. Frediani, K. Ruud, and S. Coriani, Theor. Chim. Acc. 119, 231 (2008).

${ }^{29}$ S. Coriani, C. Hättig, P. Jørgensen, and T. Helgaker, J. Chem. Phys. 113, 3561 (2000); T. Kjærgaard, B. Jansík, P. Jørgensen, S. Coriani, and J. Michl, J. Phys. Chem. A 111, 11278 (2007).

${ }^{30}$ T. Kjærgaard, P. Jørgensen, A. J. Thorvaldsen, P. Salek, and S. Coriani, J. Chem. Theory Comput. 5, 1997 (2009).

${ }^{31}$ Y. Honda, M. Hada, M. Ehara, H. Nakatsuji, and J. Michl, J. Chem. Phys. 123, 164113 (2005)

${ }^{32}$ P. Štěpánek and P. Bouř, J. Comput. Chem. 34, 1531 (2013).

${ }^{33}$ M. Seth, T. Ziegler, A. Banerjee, J. Autschbach, S. J. A. van Gisbergen, and E. J. Baerends, J. Chem. Phys. 120, 10942 (2004).

${ }^{34}$ T. Kjærgaard, K. Kristensen, J. Kauczor, P. Jørgensen, S. Coriani, and A. J. Thorvaldsen, J. Chem. Phys. 135, 024112 (2011).

${ }^{35}$ P. Norman, D. Bishop, H. J. Aa. Jensen, and J. Oddershede, J. Chem. Phys. 115, 10323 (2001).

${ }^{36}$ P. Norman, D. Bishop, H. J. Aa. Jensen, and J. Oddershede, J. Chem. Phys. 123, 194103 (2005).

${ }^{37}$ H. Solheim, K. Ruud, S. Coriani, and P. Norman, J. Chem. Phys. 128, 094103 (2008); T. Fahleson, J. Kauczor, P. Norman, and S. Coriani, Mol. Phys. 111, 1401 (2013).

${ }^{38}$ H. Solheim, K. Ruud, S. Coriani, and P. Norman, J. Phys. Chem. A 112, 9615 (2008). 
${ }^{39}$ P. Norman, K. Ruud, and T. Helgaker, J. Chem. Phys. 120, 5027 (2004); A. Jiemchooroj and P. Norman, ibid. 126, 134102 (2007); A. Jiemchooroj, U. Ekström, and P. Norman, ibid. 127, 165104 (2007).

${ }^{40}$ U. Ekström, and P. Norman, Phys. Rev. A 74, 042722 (2006); U. Ekström, P. Norman, V. Carravetta, and H. Ågren, Phys. Rev. Lett. 97, 143001 (2006)

${ }^{41}$ P. Norman, A. Jiemchooroj, and B. E. Sernelius, J. Chem. Phys. 118, 9167 (2003); A. Jiemchooroj, P. Norman, and B. E. Sernelius, ibid. 125, 124306 (2006).

${ }^{42}$ K. Kristensen, J. Kauczor, A. J. Thorvaldsen, P. Jørgensen, T. Kjærgaard, and A. Rizzo, J. Chem. Phys. 134, 214104 (2011).

${ }^{43}$ M. Krykunov, M. Seth, T. Ziegler, and J. Autschbach, J. Chem. Phys. 127, 244102 (2007).

${ }^{44}$ K.-M. Lee, K. Yabana, and G. F. Bertsch, J. Chem. Phys. 134, 144106 (2011).

${ }^{45}$ DALTON, a molecular electronic structure program, Release Dalton2013, 2013, see http://daltonprogram.org; K. Aidas, C. Angeli, K. L. Bak, V. Bakken, R. Bast, L. Boman, O. Christiansen, R. Cimiraglia, S. Coriani, P. Dahle, E. K. Dalskov, U. Ekström, T. Enevoldsen, J. J. Eriksen, P. Ettenhuber, B. Fernández, L. Ferrighi, H. Fliegl, L. Frediani, K. Hald, A. Halkier, C. Hättig, H. Heiberg, T. Helgaker, A. C. Hennum, H. Hettema, E. Hjertenæs, S. Høst, I.-M. Høyvik, M. F. Iozzi, B. Jansik, H. J. Aa. Jensen, D. Jonsson, P. Jørgensen, J. Kauczor, S. Kirpekar, T. Kjærgaard, W. Klopper, S. Knecht, R. Kobayashi, H. Koch, J. Kongsted, A. Krapp, K. Kristensen, A. Ligabue, O. B. Lutnæs, J. I. Melo, K. V. Mikkelsen, R. H. Myhre, C. Neiss, C. B. Nielsen, P. Norman, J. Olsen, J. M. H. Olsen, A. Osted, M. J. Packer, F. Pawlowski, T. B. Pedersen, P. F. Provasi, S. Reine, Z. Rinkevicius, T. A. Ruden, K. Ruud, V. Rybkin, P. Salek, C. C. M. Samson, A. Sánchez de Merás, T. Saue, S. P. A. Sauer, B. Schimmelpfennig, K. Sneskov, A. H. Steindal, K. O. Sylvester-Hvid, P. R. Taylor, A. M. Teale, E. I. Tellgren, D. P. Tew, A. J. Thorvaldsen, L. Thøgersen, O. Vahtras, M. A. Watson, D. J. D. Wilson, M. Ziolkowski, and H. Ågren, "The Dalton quantum chemistry program system," WIREs Comput. Mol. Sci. 2013 (published online).

${ }^{46}$ J. Kauczor, P. Jørgensen, and P. Norman, J. Chem. Theory Comput. 7, 1610 (2011).

${ }^{47}$ J. M. L. Martin and P. R. Taylor, Chem. Phys. Lett. 248, 336 (1996).

${ }^{48}$ J. Gauss and J. F. Stanton, J. Phys. Chem. A 104, 2865 (2000).

${ }^{49}$ A. D. Becke, J. Chem. Phys. 98, 5648 (1993); P. J. Stephens, F. J. Devlin, C. F. Chabalowski, and M. J. Frisch, J. Phys. Chem. 98, 11623 (1994).

${ }^{50}$ C. Lee, W. Yang, and R. G. Parr, Phys. Rev. B 37, 785 (1988).

${ }^{51}$ R. A. Kendall, Th. H. Dunning, Jr., and R. J. Harrison, J. Chem. Phys. 96, 6796 (1992)
${ }^{52}$ M. J. Frisch, G. W. Trucks, H. B. Schlegel et al., GAUSSIAN 09, Revision C.01, Gaussian, Inc., Wallingford, CT, 2010.

${ }^{53}$ D. E. Woon and Th. H. Dunning, Jr., J. Chem. Phys. 103, 4572 (1995).

${ }^{54}$ J. P. Perdew, K. Burke, and M. Ernzerhof, Phys. Rev. Lett. 77, 3865 (1996); 78, 1396 (1997) (Erratum).

${ }^{55}$ A. D. Becke, Phys. Rev. A 38, 3098 (1988).

${ }^{56}$ T. Yanai, D. P. Tew, and N. C. Handy, Chem. Phys. Lett. 393, 51 (2004).

${ }^{57}$ A. D. Becke, J. Chem. Phys. 98, 1372 (1993).

${ }^{58}$ O. Christiansen, H. Koch, and P. Jørgensen, Chem. Phys. Lett. 243, 409 (1995).

59 J. Cížek, Adv. Chem. Phys. 14, 35 (1969); G. D. Purvis III and R. J. Bartlett, J. Chem. Phys. 76, 1910 (1982); G. E. Scuseria, C. L. Janssen, and H. F. Schaefer III, ibid. 89, 7382 (1988); G. E. Scuseria and H. F. Schaefer III, ibid. 90, 3700 (1989).

${ }^{60}$ O. Christiansen, H. Koch, and P. Jørgensen, J. Chem. Phys. 103, 7429 (1995); H. Koch, O. Christiansen, P. Jørgensen, A. Sánchez de Merás, and T. Helgaker, ibid. 106, 1808 (1997).

${ }^{61}$ E. R. Davidson and A. A. Jarzecki, Chem. Phys. Lett. 285, 155 (1998).

${ }^{62}$ L. Serrano-Andrés, M. Merchán, I. Nebot-Gil, R. Lindh, and B. O. Roos, J. Chem. Phys. 98, 3151 (1993).

${ }^{63}$ See supplementary material at http://dx.doi.org/10.1063/1.4869849 for tables of the calculated, lowest dipole-allowed excitation energies for ethene, benzene, para-benzoquinone, and ethanol; table of the basis-set dependence of NSCD and NSOR in ethene; table of comparison between standard response theory results for NSOR with those of CPP response theory, in the off-resonant spectral regions; tables of calculated MCD, Faraday OR, NSCD, and NSOR signals in ethene, benzene, para-benzoquinone, and ethanol; illustrations of the calculated basis-set convergence of the electric-dipole transition moments to the lowest excited states of ethene, dependence of the oscillator strengths of the same on the used electron correlation method, excitation energies for benzene, para-benzoquinone, and ethanol, and ${ }^{17} \mathrm{OSCD}$ and ${ }^{17} \mathrm{OSOR}$ in para-benzoquinone, as well as MCD, Faraday OR, NSCD, and NSOR in ethanol, as obtained with various computational methods.

${ }^{64}$ N. Nakashima, H. Inoue, M. Sumitami, and K. Yoshihara, J. Chem. Phys. 73, 5976 (1980).

${ }^{65}$ A. Hiraya and K. Shobatake, J. Chem. Phys. 94, 7700 (1991).

${ }^{66}$ A. Kuboyama, S. Matsuzaki, H. Tagagi, and H. Arano, Bull. Chem. Soc. Jpn. 47, 1604 (1974).

${ }^{67}$ A. R. Meier and G. H. Wagnière, Chem. Phys. 113, 287 (1987).

${ }^{68}$ I. Schapiro, K. Sivalingam, and F. Neese, J. Chem. Theory Comput. 9, 3567 (2013).

${ }^{69}$ L. R. Ingersoll and D. H. Liebenberg, J. Opt. Soc. Am. 44, 566 (1954). 